\title{
Understanding Hollow Metal Oxide Nanomaterial Formation with in situ Transmission Electron Microscopy
}

\author{
Lei Yu ${ }^{1}$, Ruixin $\mathrm{Han}^{1}$, Xiahan Sang ${ }^{2}$, Jue Liu ${ }^{3}$, Amita Patel ${ }^{1}$, Katharine Page ${ }^{3}$ and Beth S. Guiton ${ }^{1}$ \\ 1. Department of Chemistry, University of Kentucky, Lexington, KY 40506 \\ 2. Center for Nanophase Materials Sciences, Oak Ridge National Laboratory, Oak Ridge, TN 37831 \\ 3. Chemical and Engineering Materials Division, Oak Ridge National Laboratory, Oak Ridge, TN 37831
}

Nanomaterials have been studied intensely for several decades, in large part for potential applications such as catalysis, energy storage and sensors, [1-3] which rely on their high surface area. Hollow nanostructures with their especially high surface areas, low densities and large capacities are particularly promising, and the properties of hollow nanomaterials are often found to be favorable as compared to their solid counterparts.[4] Hollow iron oxides are of particular interest, having already been shown to perform well as photocatalysts, super capacitors, and anodes for lithium ion batteryies.[5-7] In order to fully exploit the potential of these hollow iron oxide nanomaterials, however, it is critical to understand and control the crystal structure (for the many different iron oxide phases) and morphology, and to understand the resulting structure-property relationships. Much work has been done to understand mechanisms leading to hollow nanostructures, such as the Kirkendall effect, and Oswald ripening, but these mechanisms alone cannot account for every scenario. Here we discuss the in situ characterization of series of phase transformations as solid $\mathrm{FeOOH}$ nanorods evolves to produce hollow nanostructures of $\alpha-\mathrm{Fe}_{2} \mathrm{O}_{3}, \gamma-\mathrm{Fe}_{2} \mathrm{O}_{3}, \mathrm{Fe}_{3} \mathrm{O}_{4}$ and $\mathrm{FeO}$. In situ observation of a single individual $\mathrm{FeOOH}$ nanorod in the transmission electron microscopy (TEM), shows a new mechanism for the formation of a hollow capsule

$\beta-\mathrm{FeOOH}$ nanorods and nanowires for this study were all grown via hydrothermal synthesis. The as grown nanomaterials were characterized by X-ray diffraction (XRD) to identify the crystal structure, and were then prepared for in situ heating in the TEM. As the temperature was ramped, the morphological progression of a solid single-crystal to form a porous polycrystalline structure, and finally a hollow single-crystalline nanorod, was observed within an individual $\beta$-FeOOH nanorod (Figure 1). To further understand the reaction mechanism we quenched nanorods at selected times during the phase transformation processes, and performed selected area electron diffraction and electron energy loss spectroscopy to identify the iron oxide species at different heating stages. The structure was seen to evolve through a series of phase transformations, from $\beta$-FeOOH to $\beta-\mathrm{Fe}_{2} \mathrm{O}_{3}, \gamma-\mathrm{Fe}_{2} \mathrm{O}_{3}, \mathrm{Fe}_{3} \mathrm{O}_{4}$ and $\mathrm{FeO}$, accompanied by the solid-to-hollow morphological transition. However, the phase transformations in ambient environment evolve from $\beta-\mathrm{FeOOH}$ to $\alpha-\mathrm{Fe}_{2} \mathrm{O}_{3}$, accompanied by the same morphological transition as in TEM. Complementary energy dispersive x-ray spectroscopy, in situ XRD and thermal gravimetric analysis measurements were also performed.

By way of comparison we performed similar in situ heating experiments on $\gamma$-MnOOH nanowires. In this case the nanowire is observed to become thinner rather than forming a hollow tube (Figure 2), suggesting that the crystal structure of the starting species must be a crucial determining factor in the eventual morphology. Heating $\beta$-FeOOH nanowires (as opposed to nanorods) in TEM leads to hollow iron oxide tubes. 


\section{References:}

[1] G. Prieto et al, Chem. Rev. 116 (2016) p. 14056.

[2] L. Zhou et al, Adv. Mater. (2017) p.1202914-1.

[3] A. Rao et al, ACS Appl. Mater. Interfaces 9 (2017) p. 2634.

[4] W. Wei et al, Journal of Power Sources 238 (2013) p. 376.

[5] X. Qu et al, ACS Nano 4 (2010) p.1732.

[6] P. Yang et al, Nano Lett. 14 (2014) p.731.

[7] L. Zhang et al, Adv. Energy Mater. 4 (2014) p.1501333-1.

[8] The Authors acknowledge funding from National Science Foundation under award numbers OIA 1355438 and DMR 145515. Work by J. L. and K.P. was supported through the U.S. Department of Energy, Office of Science, Office of Basic Energy Sciences, Early Career Research Program award KC040602, under contract number DE-AC05-00OR22725. Microscopy performed as part of a user project at Oak Ridge National Laboratory's Center for Nanophase Materials Sciences, which is a U.S. Dept. of Energy Office of Science User Facility.

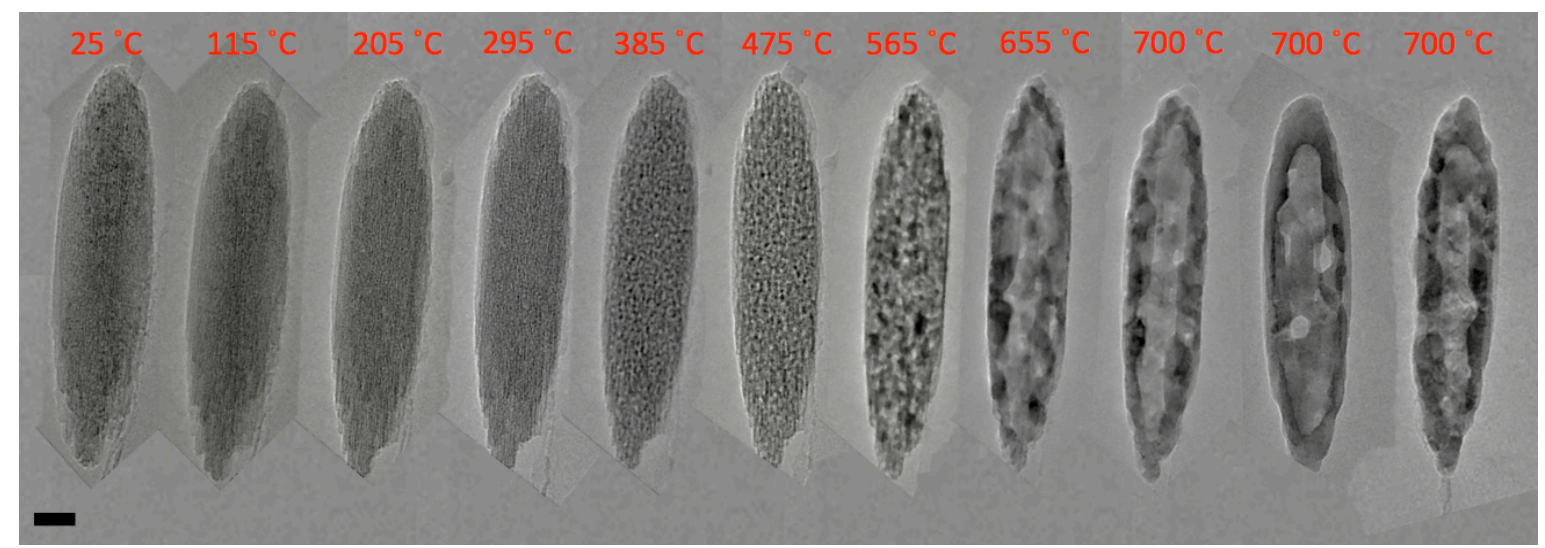

Figure 1. Snapshots from a movie recorded during heating of a $\beta$-FeOOH nanorod in the TEM, showing hollow nanorod formation. Scale bar equals $50 \mathrm{~nm}$.

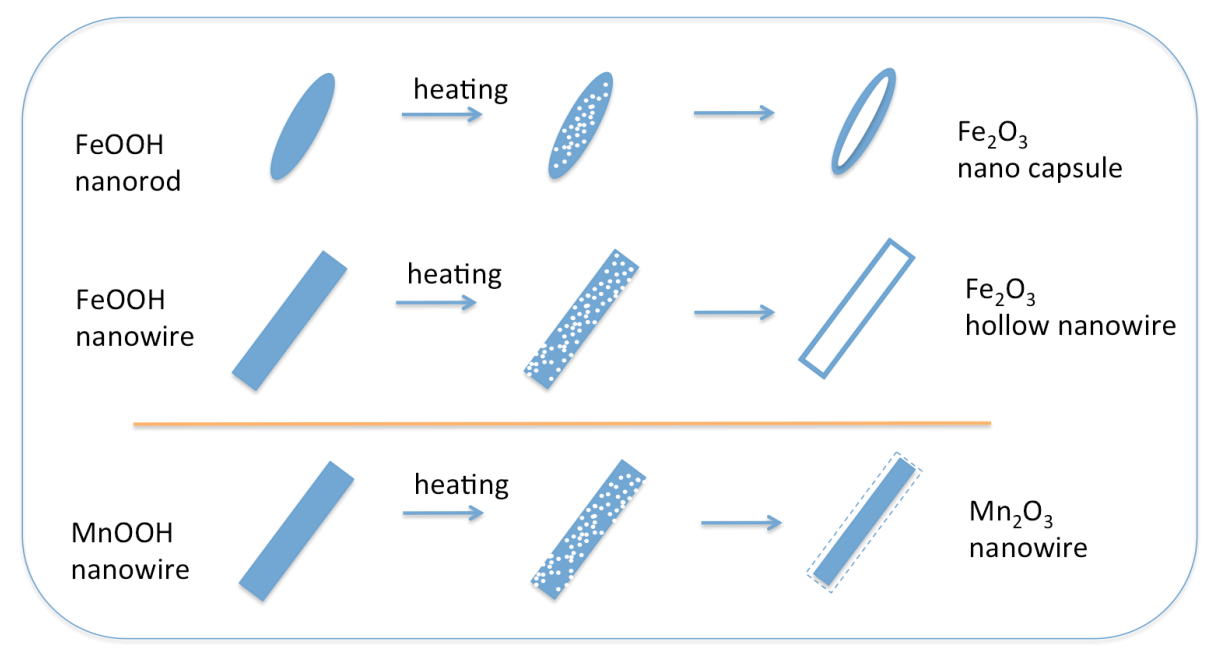

Figure 2. Schematics of morphological evolution of metal oxyhydroxide nanorods and nanowires 